\title{
The Prognostic Value of T1 Bladder Cancer Substaging: A Single Institution Retrospective Study
}

\author{
V. Soukup ${ }^{a} \quad$ J. Duškováb ${ }^{\text {M. Pešla }}$ O. Čapoun $^{a} \quad$ Z. Feherováa L. Zámečník $^{a}$ \\ T. Hanuša M. Babjuk ${ }^{c}$ \\ Departments of a Urology and b Pathology, General Teaching Hospital and 1st Faculty of Medicine, \\ Charles University in Prague, and 'Department of Urology, Hospital in Motol and 2nd Faculty of Medicine, \\ Charles University in Prague, Prague, Czech Republic
}

\section{Key Words}

T1 substaging - Urothelial carcinoma of the urinary bladder · Recurrence · Progression · Disease-specific survival $\cdot$ Overall survival

\section{Abstract}

Objective: To evaluate the prognostic value of the depth of lamina propria invasion in patients with $\mathrm{T} 1$ bladder cancer.

Subjects and Methods: 200 patients were treated between the years 2002 and 2009. Tumours with depth of invasion above the muscularis mucosae level were categorised as pT1a and those with depth of invasion up to or beyond the muscularis mucosae as pT1b. Results: Categorisation for pT1a and pT1b was performed in 176 of 200 patients (88\%). In 10 patients a muscle-invasive tumour was found in retransurethral resection samples. 131 (79\%) of 166 analysed patients had pT1a tumour and 35 (21\%) had pT1b tumour. During the follow-up, in 101 (61\%) patients the tumour had recurred and in $27(16.3 \%)$ the tumour had progressed. Of all the investigated parameters, T1 substaging ( $p<0.0001)$, grade $(p=0.0003)$ and the number of bacillus CalmetteGuérin instillations $(p=0.0490)$ were significant in predicting progression. The only significant factor for disease-specific survival was $T 1$ substaging in univariable $(p=0.0008)$ and

\section{KARGER}

(c) 2014 S. Karger AG, Basel

0042-1138/14/0922-0150\$39.50/0

E-Mail karger@karger.com

www.karger.com/uin multivariable (hazard ratio 4.407$)$ analysis. T1 substaging $(p=0.0149)$ and tumour multiplicity $(p=0.0448)$ have a statistically significant prognostic value with respect to overall survival. Conclusions: Deep invasion of the lamina propria is a significant adverse prognostic factor for tumour progression, disease-specific survival and overall survival.

(c) 2014 S. Karger AG, Basel

\section{Introduction}

Invasion of the lamina propria, tumour grade and carcinoma in situ (CIS) represent significant risk factors for the progression of non-muscle-invasive bladder cancer $[1,2]$. Despite these risk factors, predicting T1 bladder cancer behaviour remains difficult [3]. The depth of invasion of the subepithelial connective tissue may help separate patients with different clinical outcomes. The existence of muscularis mucosae (MM) was described 30 years ago as both a continuous or interrupted layer, or as only thin wisps of smooth muscle fibres located a third to halfway between the epithelium and the detrusor muscle at the same level where larger arteries can be seen [4]. According to this concept, true lamina propria is the connective tissue between the epithelium and the MM, and 
submucosa is the connective tissue between the MM and the muscularis propria. Therefore it seems that the MM could be used as a landmark for more precise staging and for subcategorisation of patients with T1 tumours.

Invasion depth of the lamina propria has been associated with an increased risk of disease progression and death [5-9]. Despite this fact, T1 bladder cancer substaging has not been recommended in either clinical guidelines or classification systems for stages $[10,11]$. The purpose of the present study was to assess in a prospective manner the routine clinical use of identifying the MM and its prognostic value as a landmark in T1 bladder cancer substaging. We focused on the usefulness and applicability of this prognostic factor in daily clinical practice.

\section{Subjects and Methods}

Between the years 2002 and 2009, 200 patients with T1 bladder cancer were treated at our institution. Among them, 141 (71\%) cases were primary and 59 (29\%) cases were recurrent with previous noninvasive ( $\mathrm{Ta}$ ) tumours. A transurethral resection (TUR) of bladder urothelial cell cancer was performed using a standard technique, removing all of the visually identifiable disease and the biopsy from the tumour base. Random biopsies of normal mucosa were not performed, whereas cold cup biopsies were taken from suspicious areas. The surgery was finished with an endoscopic inspection using a $70^{\circ}$ telescope, finding no visible tumour lesion. A second resection was performed if the TUR was considered to be incomplete and/or the detrusor muscle was absent from the pathology specimen. Tumours were graded according to the World Health Organization system [12].

Based on the depth of the invasion, T1 tumours were subclassified into pT1a tumours (tumour invasion to the lamina propria above the level of the MM) and pT1b tumours (cells invading up to or beyond the MM without involving the muscularis propria). When the MM was not identifiable, large vessels accompanying the MM provided evidence of the presumed level. Pathological T1 bladder cancer substaging has been performed as a routine procedure by several different pathologists over an extended period.

The median follow-up time was 3.13 years $(0.1-10.5$ years). Patients were initially examined at 3-month intervals using cystoscopy and urine cytology. Patients without recurrences for the first 2 years were seen at 6-month intervals for 5 years after the operation and then annually. Follow-up examinations began by the date of the TUR of the bladder tumour and lasted until the time of death or until the last visit. Recurrence was defined as a new, non-muscle-invasive tumour occurring over 3 months after the initial operation. Disease progression was defined as the occurrence of a muscle-invasive tumour (stage T2 or higher) or metastatic disease. Treatment consisted of repeated TUR with or without intravesical administration of bacillus Calmette-Guérin (BCG) therapy or intravesical chemotherapy. One immediate intravesical instillation of chemotherapy was not given to any patient during the study period.

Prognostic Value of T1 Bladder Cancer Substaging
Table 1. Characteristics of the analysed patients

\begin{tabular}{ll}
\hline $\begin{array}{l}\text { Females/males } \\
\text { Age, years }\end{array}$ & $45 / 121$ \\
$\quad$ Median & \\
$\quad$ Range & 68.83 \\
T1a/T1b & $17.55-86.94$ \\
Primary/recurrent & $131 / 35$ \\
G1/G2/G3 & $114 / 52$ \\
CIS (yes/no) & $35 / 84 / 47$ \\
Single/multiple & $19 / 147$ \\
Size $(<3$ cm/>3 cm) & $66 / 100$ \\
re-TUR (yes/no) & $80 / 86$ \\
BCG (yes/no) & $59 / 107$ \\
MMC (yes/no) & $36 / 130$ \\
\end{tabular}

$\mathrm{BCG}=$ Intravesical treatment with BCG; $\mathrm{MMC}=$ intravesical treatment with mitomycin $\mathrm{C}$; re-TUR = second transurethral resection 4-6 weeks after transurethral resection.

For statistical analysis, the following baseline variables were considered for their prognostic value: stage $(\mathrm{pT} 1 \mathrm{a} \times \mathrm{pT} 1 \mathrm{~b})$, grade (G1, G2, G3), multiplicity (multiple $\times$ solitary), presence of CIS, tumour size $(<3 \mathrm{~cm} \times>3 \mathrm{~cm}$ in diameter), performance of re-TUR, administration of BCG therapy, number of BCG instillations, length of BCG therapy, administration of intravesical chemotherapy, number of chemotherapy instillations, length of intravesical chemotherapy, age and gender. Recurrence-free status, progression-free status, overall and cancer-specific survival were analysed using the Kaplan-Meier method; the log-rank test was used to compare curves for two or more groups $[13,14]$. For all analyses $\mathrm{p}<0.05$ was considered statistically significant. Patients' death of other causes, or their being lost to further examination, was censored at the date of the last follow-up. Cox's proportional hazards model was used for the multivariate analysis of prognostic parameters to estimate relative risk and to select independent prognostic factors. The results were expressed as a hazard ratio, which was derived from the estimated regression coefficients with their $95 \%$ confidence intervals [15].

\section{Results}

Categorisation into pT1a and pT1b could be performed in 176 of 200 patients (88\%). Muscle-invasive tumour was found in re-TUR samples in 10 patients - in 2 of $37(5.4 \%)$ patients with T1a tumour and 8 of $32(25 \%)$ patients with T1b tumour. The risk of upstaging to muscle-invasive disease in $\mathrm{pT} 1 \mathrm{~b}$ patients was more than 4.5 times higher than that of $\mathrm{pT} 1 \mathrm{a}$ patients. These 10 patients were excluded from further analysis. 131 (79\%) of 166 analysed patients had a pT1a tumour and $35(21 \%)$ had a pT1b tumour. The characteristics of the analysed patients can be seen in table 1 . 


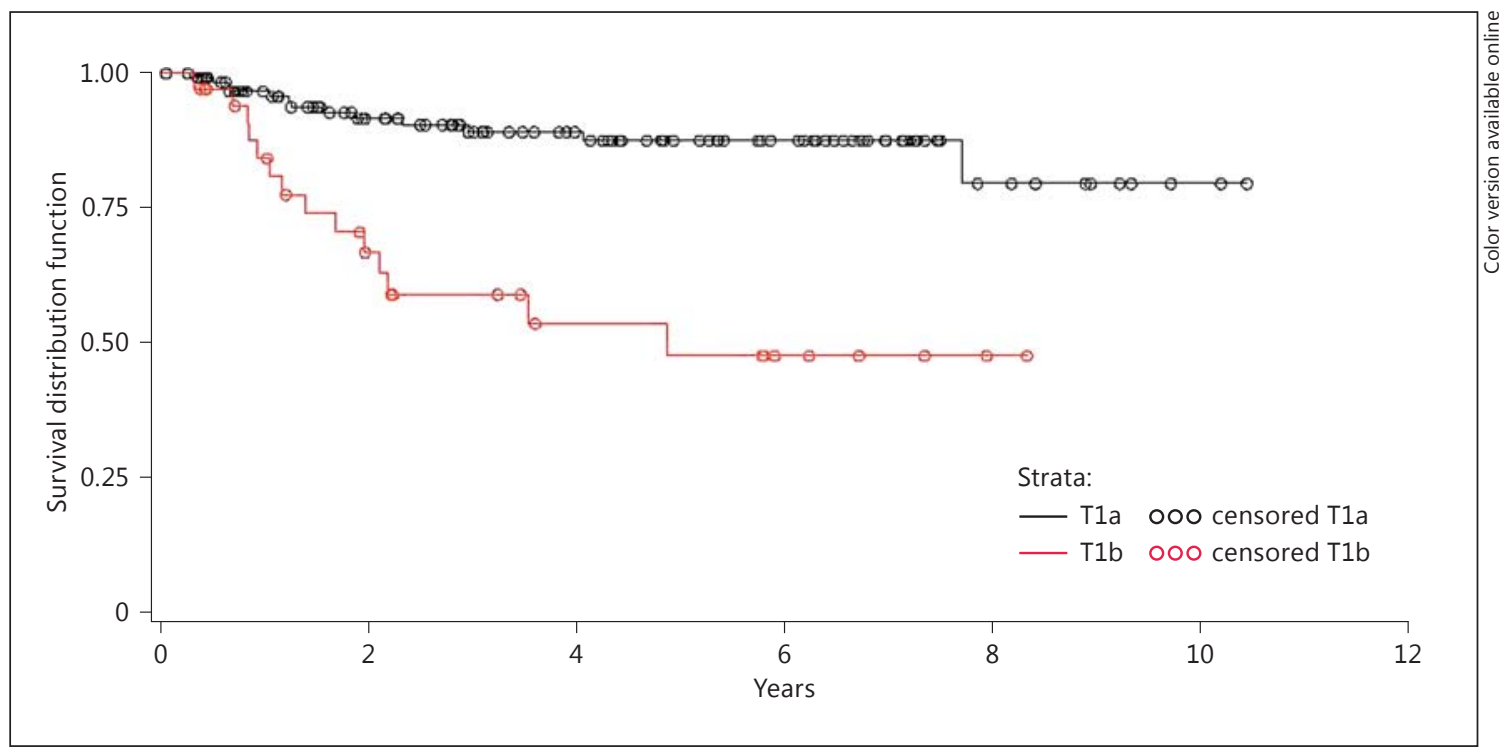

Fig. 1. Kaplan-Meier curves of percentage of patients remaining free of progression (log-rank test, $\mathrm{p}<0.0001)$. Black line: patients with T1a tumour (131 patients). Grey line (red in online version): patients with T1b tumour (35 patients).

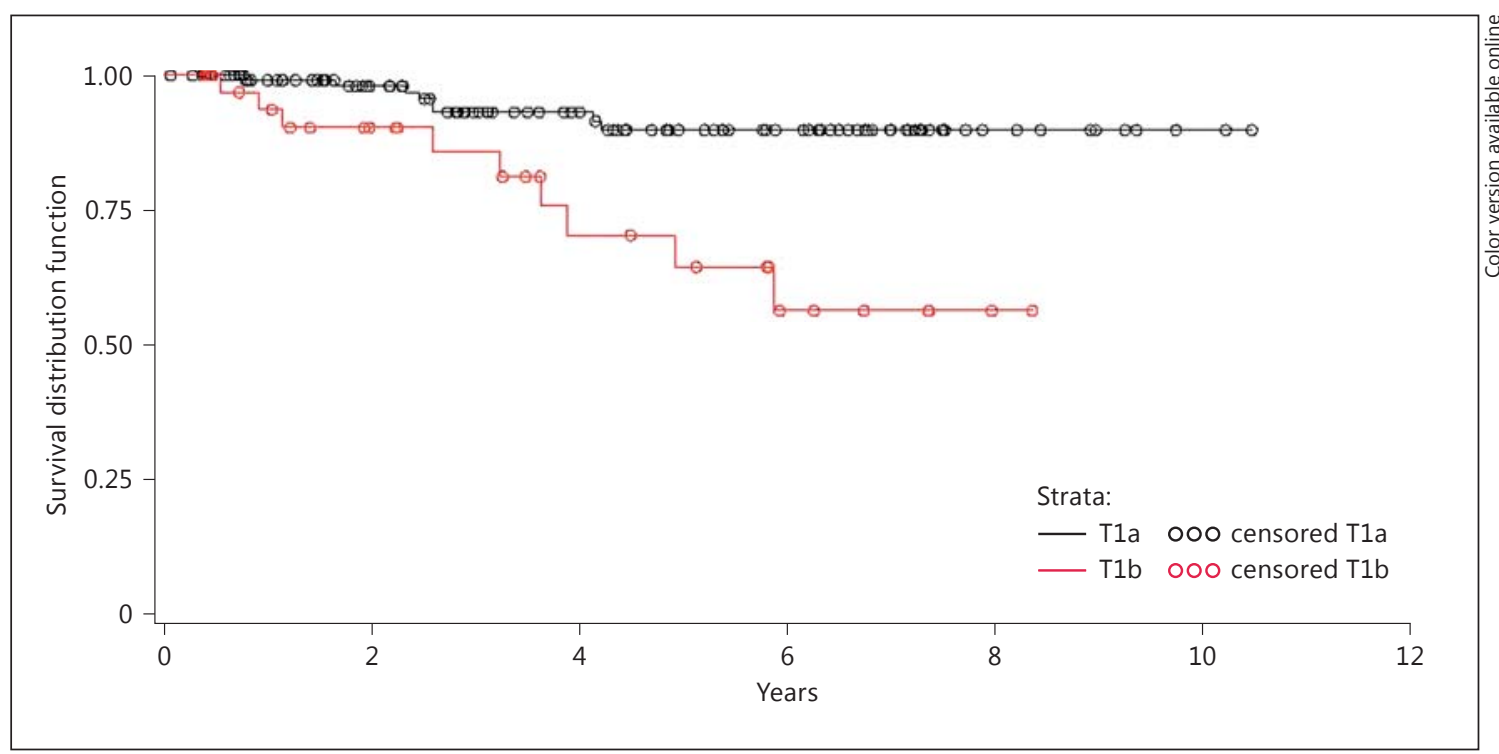

Fig. 2. Kaplan-Meier curves of disease-specific survival (log-rank test, $\mathrm{p}=0.0008$ ). Black line: patients with T1a tumour (131 patients). Grey line (red in online version): patients with T1b tumour (35 patients).

The depth of tumour invasion was significantly associated with high tumour grade $(\mathrm{p}<0.0001)$ and the presence of CIS ( $p=0.0003$ ). The relationship between T1 substaging and other standard clinical variables such as tumour size $(\mathrm{p}=0.1028)$, tumour multiplicity $(\mathrm{p}=0.9740)$, age $(\mathrm{p}=0.3171)$ and gender $(\mathrm{p}=0.1344)$ were not significant.
The level of lamina propria invasion also correlated with BCG treatment $(\mathrm{p}<0.0001)$ and performance of re-TUR $(\mathrm{p}<0.0001)$. These treatment modalities were significantly more frequent in patients with $\mathrm{T} 1 \mathrm{~b}$ tumours.

During the follow-up period, 101 (61\%) patient experienced recurrence and 27 (16.3\%) patients experienced 


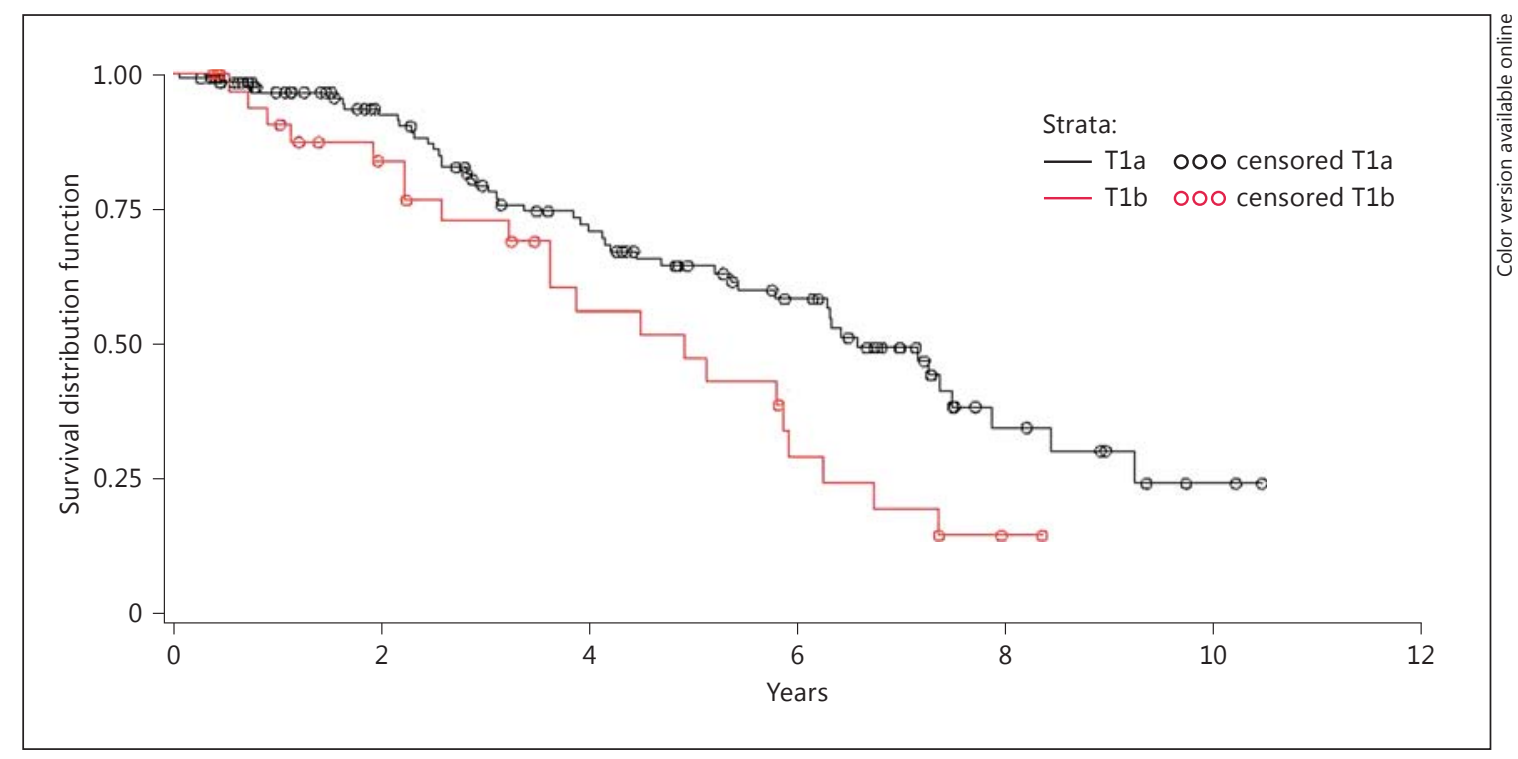

Fig. 3. Kaplan-Meier curves of overall survival (log-rank test, $\mathrm{p}=0.0149$ ). Black line: patients with T1a tumour (131 patients). Grey line (red in online version): patients with T1b tumour (35 patients).

progression, $17(10.2 \%)$ patients died due to tumour progression and 52 (31.3\%) patients died from other causes. The depth of lamina propria invasion was not discriminative in predicting recurrence $(\mathrm{p}=0.7234)$. With the Kaplan-Meier analyses, tumour multiplicity $(\mathrm{p}=0.0008)$, reTUR ( $p=0.0330)$, BCG instillation therapy $(\mathrm{p}=0.0328)$ and the number of BGG instillations $(\mathrm{p}=0.0094)$ were statistically significant in predicting tumour recurrence.

According to the Kaplan-Meier actuarial method, the progression-free interval was significantly different in patients with pT1a tumours compared to those with $\mathrm{pT} 1 \mathrm{~b}$ tumours ( $p<0.0001)$. Curves for progression-free estimates are plotted in figure 1 . A significant correlation was also found between progression-free survival and tumour grade $(p=0.0003)$ and the number of BCG instillations $(\mathrm{p}=0.0490)$. Multivariate analysis using the proportional hazard model (Cox's regression) identified T1 substaging and grade acting as independent predictors of tumour progression (table 2).

The only significant factor for disease-specific survival was T1 substaging $(\mathrm{p}=0.0008)$ in univariate and multivariate analysis (fig. 2, table 2 ).

T1 substaging $(\mathrm{p}=0.0149)$ and tumour multiplicity ( $\mathrm{p}=0.0448)$ have a statistically significant prognostic value with respect to overall survival (fig. 3). Independent factors for overall survival in Cox's regression analysis were T1 substaging and BCG instillation therapy (table 2).

Prognostic Value of T1 Bladder Cancer Substaging
Additional analysis was performed on the subgroup of 116 primary bladder tumours. T1 substaging was found to be a significant prognostic factor with respect to tumour progression $(\mathrm{p}<0.0001)$, cancer-specific survival $(\mathrm{p}=0.0001)$ and overall survival $(\mathrm{p}=0.0002)$ (KaplanMeier curves are not shown). In Cox's regression analysis, T1 substaging was an independent factor for progressionfree, disease-specific and overall survival. The prognostic value of T1 substaging in the subgroup of patients with primary tumours was stronger than it was in the entire study group, where 59 (29\%) cases were recurrent with previous non-invasive ( $\mathrm{Ta}$ ) tumours (table 3 ).

\section{Discussion}

Depth of invasion within the subepithelial connective tissue in the TUR specimen has a prognostic value with respect to the risk of tumour progression [6-8, 16-22]. A distinctive prognosis can be expected for tumours with different levels of invasion [4]. The studies that have addressed the subject, however, have not found a reliable method for subdividing the level of invasion.

The most common method of T1 bladder cancer substaging has been realized by means of the concept of the MM and the associated large blood vessels [4]. Several systems of T1 bladder cancer substaging using the MM as a landmark have been described. It has been proposed 
Table 2. Multivariate Cox's proportional hazards model analysis - independent prognostic factors for the prediction of tumour recurrence, tumour progression, disease-specific and overall survival in the whole study population $(n=166)$

\begin{tabular}{lllll}
\hline Analysis & Variable & HR & 95\% HR, CI & p value \\
\hline RFS & multiplicity & 2.315 & $1.507,3.555$ & 0.0001 \\
& BCG therapy & 2.192 & $1.269,3.785$ & 0.0049 \\
\hline PFS & T1 substaging & 3.562 & $1.532,8.282$ & 0.0032 \\
& grade & 2.515 & $1.252,5.052$ & 0.0095 \\
\hline \multirow{2}{*}{ DSS } & T1 substaging & 4.407 & $1.699,11.430$ & 0.0023 \\
\hline OS & age & 3.925 & $2.257,6.826$ & $<0.0001$ \\
& T1 substaging & 3.109 & $1.615,5.986$ & 0.0007 \\
& BCG therapy & 2.469 & $1.214,5.021$ & 0.0126 \\
\hline
\end{tabular}

$\mathrm{CI}=$ Confidence interval; DSS = disease-free survival; $\mathrm{HR}=$ hazard ratio; $\mathrm{OS}=$ overall survival; $\mathrm{PFS}=$ progression-free survival; RFS = recurrence-free survival.

Table 3. Multivariate Cox's proportional hazards model analysis - independent prognostic factors for the prediction of tumour recurrence, tumour progression, disease-specific and overall survival in a subset of patients with the primary tumour $(\mathrm{n}=114)$

\begin{tabular}{llrlr}
\hline Analysis & Variable & \multicolumn{1}{l}{ HR } & 95\% HR, CI & p value \\
\hline RFS & multiplicity & 1.712 & $1.047,2.801$ & 0.0322 \\
\hline PFS & T1 substaging & 12.520 & $3.621,43.285$ & $<0.0001$ \\
& grade & 3.741 & $1.493,9.374$ & 0.0049 \\
& re-TUR & 3.855 & $1.280,11.610$ & 0.0164 \\
\hline DSS & T1 substaging & 13.769 & $3.846,49.290$ & $<0.0001$ \\
& re-TUR & 4.614 & $1.379,15.431$ & 0.0131 \\
\hline OS & age & 4.837 & $2.347,9.969$ & $<0.0001$ \\
& T1 substaging & 7.297 & $3.172,16.790$ & $<0.0001$ \\
& BCG therapy & 4.329 & $1.789,10.478$ & 0.0012 \\
\hline
\end{tabular}

$\mathrm{CI}=$ Confidence interval; DSS = disease-free survival; HR = hazard ratio; $\mathrm{OS}=$ overall survival; $\mathrm{PFS}=$ progression-free survival; re-TUR $=$ second transurethral resection $4-6$ weeks after transurethral resection; RFS = recurrence-free survival.

that the MM could be used to differentiate the levels of invasion by pT1a - invasion of connective tissue superficial to the level of the MM, pT1b - invasion to the level of the MM, and pT1c - invasion through the level of the MM but superficial to the muscularis propria $[5,8,9,18,23]$. A second proposal divides T1 patients into two populations only. Tumours superficial to the MM or reaching the MM but not passing through it were classified as
pT1a, and tumours passing through the MM and invading the submucosa were considered to be pT1b $[16,21$, 24]. A third proposal subclassifies $\mathrm{T} 1$ tumours as either with MM invasion (pT1b) or without (pT1a) $[6,7,17,20$, $25,26]$. The lack of uniformity between these systems of substaging could make data comparison difficult.

Another significant problem is that the MM could not be identified in every patient. Substaging rates range from 58 to $100 \%$ in various publications $[6,9,16,17,20,21,23]$, which argues against the usefulness of the MM as a tool for $\mathrm{T} 1$ substaging [23]. We were able to subclassify T1 tumours in $88 \%$ of the patients. We consider the percentage to be high because 59 patients (29\%) had recurrent tumours, in whom a previous TUR could have damaged the submucosa and made interpretation more difficult.

The inability to determine the MM in every patient was the reason for creating substaging systems which are not based on MM identification. In a study by Cheng et al. [27], the depth of invasion was measured with a micrometre, and it was stated that an invasion depth of $1.5 \mathrm{~mm}$ from the basement membrane should be the optimal threshold for indicating advanced-stage cancer. Another method of substaging was proposed recently by van Rhijn et al. $[22,28]$ and discerns T1-microinvasive (T1m) and T1-extensive-invasive (T1e) tumours. The definition of $\mathrm{T} 1 \mathrm{~m}$ was a single focus of lamina propria invasion with a diameter $\leq 0.5 \mathrm{~mm}$ (within one high-power field, objective $\times 40$ ). Specimens showing a larger area with invasion or multiple microinvasive areas were considered T1e. This new substaging was possible in $100 \%$ of the evaluated patients.

Despite the fact that the level of invasion in T1 tumours has prognostic value, none of the substaging systems mentioned above have became a component of the TNM bladder cancer classification [11]. There is a distinct possibility that none of them are easy enough to be performed as a part of everyday clinical practice. The new substaging system (T1m and T1e) appears to be more user-friendly, but the promising recently published results have to be confirmed in prospective studies, which may concur that this substaging system has the potential to be incorporated in future TNM classifications [22].

The prognostic value of T1 substaging has previously been reported [5-9, 16-22]. We found that the extent of lamina propria invasion is a relevant prognostic factor for tumour progression, disease-specific survival and overall survival in uni- and multivariable analyses. This observation is in concordance with previous reports $[5-9,16-$ $22]$. The depth of lamina propria invasion has been significant in multivariable analyses for overall survival in 
three studies using the substaging system based on MM identification $[9,16,21]$. Recurrence rates and recurrence-free intervals did not differ significantly for either group in our study, which was also in agreement with the results published previously $[7,8,18,24,25,28]$.

Retrospective analysis of archived T1 tumour samples was used in the majority of published studies $[5-8,16-25$, $27,28]$. In our present study, the evaluation of the depth of tumour invasion was done prospectively immediately after the TUR by several pathologists as part of the routine pathological report. We consider this fact to be the strength of our study. The study limitations include a low number of evaluated patients, a short follow-up, a retrospective evaluation of patients' outcome and treatment heterogeneity. The second TUR was performed in only 69 of 176 patients. The indication of re-TUR during the study period was suspected incompleteness of the original TUR and/or absence of the detrusor muscle in the pathology specimen. This approach followed previous guidelines but is not standard at the present time when a re-TUR is recommended in all T1 patients [10, 29]. Due to financial limits, intravesical BCG therapy was given to only 36 patients with a high risk of progression. This is the reason why the administration of intravesical therapy, the number of instillations and the length of intravesical therapy were tested as variables in the statistical analysis. A significant association between BCG instillation therapy and tumour grade $(\mathrm{p}<0.0001)$, presence of concomitant CIS ( $\mathrm{p}<0.0001)$ and depth of tumour invasion $(\mathrm{p}<$ 0.0001 ) was found: 12 patients out of 131 with pTla tu- mours (9\%) and 20 patients out of 35 with pT1b tumours (57\%) were treated with BCG. Despite this fact, our data argue that tumours invading up to or beyond the MM have a higher risk of progression than tumours that have invaded only above the level of the MM. This underscores the importance of stating this finding in the pathology report and supports the usefulness of adopting a routine assessment of the level of lamina propria invasion.

Whether or not patients with an initial diagnosis of a deep T1 tumour should be followed up and treated differently than those with superficial T1 tumours remains controversial, and prospective studies are needed not only to determine that the depth of $\mathrm{T} 1$ invasion has an independent prognostic value, but also to define the optimal therapy for patients with stage $\mathrm{pT} 1 \mathrm{~b}$ tumours.

In conclusion, deep invasion of the lamina propria could be a significant adverse prognostic factor for tumour progression, disease-specific survival and the overall survival of patients with T1 bladder cancer. Therefore, we support the suggestion that $\mathrm{T} 1$ substaging should be a routine part of any histopathological report when possible. However, prospective, randomized studies evaluating different treatment options for patients with different extents of lamina propria invasion need to be performed.

\section{Acknowledgements}

This study was supported by projects TIP ČR FR-TI3/666 and IGA NT/12205-5.

\section{References}

1 Sylvester RJ, van der Meijden AP, Oosterlinck $\mathrm{W}$, et al: Predicting recurrence and progression in individual patients with stage Ta T1 bladder cancer using EORTC risk tables: a combined analysis of 2596 patients from seven EORTC trials. Eur Urol 2006;49:466-475.

-2 Altieri VM, Castellucci R, Palumbo P, Verratti V, Sut M, Olivieri R, Manco R, Ricciardulli S, Nicolai M, Criniti P, Tenaglia RL: Recurrence and progression in non-muscleinvasive bladder cancer using EORTC risk tables. Urol Int 2012;89:61-66.

3 Reljic A, Trnski D: We abandoned 'superficial bladder cancer': we accepted 'non-muscle-invasive bladder cancer', but ... - on occasion of the 10th anniversary of the first EAU Guidelines on bladder cancer. Urol Int 2012; 88:486-487.
4 Dixon JS, Gosling JA: Histology and fine structure of the muscularis mucosae of the human urinary bladder. J Anat 1983;136:265271.

5 Younes M, Sussman J, True LD: The usefulness of the level of the muscularis mucosae in the staging of invasive transitional cell carcinoma of the urinary bladder. Cancer 1990;66: 543-548.

6 Hasui Y, Osada Y, Kitada S, et al: Significance of invasion to the muscularis mucosae on the progression of superficial bladder cancer. Urology 1994;43:782-786.

-7 Holmang S, Hedelin H, Anderstrom C, et al: The importance of the depth of invasion in stage T1 bladder carcinoma: a prospective cohort study. J Urol 1997;157:800-803.
8 Smits G, Schaafsma E, Kiemeney L, et al: Microstaging of pT1 transitional cell carcinoma of the bladder: identification of subgroups with distinct risks of progression. Urology 1998;52:1009-1013.

9 Hermann GG, Horn T, Steven K: The influence of the level of lamina propria invasion and the prevalence of p53 nuclear accumulation on survival in stage T1 transitional cell bladder cancer. J Urol 1998;159:91-94.

10 Babjuk M, Oosterlinck W, Sylvester R, et al: EAU Guidelines on non-muscle-invasive urothelial carcinoma of the bladder. Eur Urol 2011;59:997-1008

11 Sobin DH, Wittekind C (eds): TNM Classification of Malignant Tumours, ed. 6. New York, Wiley-Liss, 2002, pp 199-202. 
12 Mostofi FK, Sobin LH, Torloni H: Histopathological typing of urinary bladder tumors; in Mostofi FK, Sobin LH, Torloni H (eds): International Classification of Tumors. Geneva, WHO, 1973.

13 Kaplan E, Meier P: Nonparametric estimation from incomplete observation. J Am Stat Assoc 1958;53:457-481.

14 Mantel N: Evaluation of survival data and two new rank order statistics arising in its consideration. Cancer Chemother Rep 1966;50: 163-170.

15 Cox DR: Regression models and life-tables. J R Stat Soc 1972;34:187-220.

16 Angulo JC, Lopez JI, Grignon DJ, et al: Muscularis mucosa differentiates two populations with different prognosis in stage T1 bladder cancer. Urology 1995;45:47-53.

17 Bernardini S, Billerey C, Martin M, et al: The predictive value of muscularis mucosae invasion and p53 over expression on progression of stage T1 bladder carcinoma. J Urol 2001 165:42-46.

18 Orsola A, Trias I, Raventós CX, et al: Initial high-grade T1 urothelial cell carcinoma: feasibility and prognostic significance of lamina propria invasion microstaging $(\mathrm{Tl} \mathrm{a} / \mathrm{b} / \mathrm{c})$ in BCG-treated and BCG-non-treated patients. Eur Urol 2005;48:231-238.
19 Andius P, Johansson SL, Holmang S: Prognostic factors in stage T1 bladder cancer: tumor pattern (solid or papillary) and vascular invasion more important than depth of invasion. Urology 2007;70:758-762.

20 Mhawech-Fauceglia P, Fischer G, Alvarez V $J r$, et al: Predicting outcome in minimally invasive (T1a and T1b) urothelial bladder carcinoma using a panel of biomarkers: a high throughput tissue microarray analysis. BJU Int 2007;100:1182-1187.

21 Faivre d'Arcier B, Celhay O, Safsaf A, et al: T1 bladder carcinoma: prognostic value of the muscularis mucosae invasion (T1a/T1b). A multicenter study by the French Urological Association (CCAFU). Prog Urol 2010;20: 440-449.

22 van Rhijn BW, van der Kwast TH, Alkhateeb SS, et al: A new and highly prognostic system to discern T1 bladder cancer substage. Eur Urol 2012;61:378-384.

23 Platz CE, Cohen MB, Jones MP, et al: Is microstaging of early invasive cancer of the urinary bladder possible or useful? Mod Pathol 1996;9:1035-1039.
24 Kondylis FI, Demirci S, Ladaga L, et al: Outcomes after intravesical bacillus CalmetteGuerin are not affected by substaging of high grade $\mathrm{T} 1$ transitional cell carcinoma. J Urol 2000;163:1120-1123.

25 Shariat SF, Weizer AZ, Green A, et al: Prognostic value of P53 nuclear accumulation and histopathologic features in T1 transitional cell carcinoma of the urinary bladder. Urology 2000;56:735-740.

26 Gohji K, Nomi M, Okamoto M, et al: Conservative therapy for stage T1b, grade 3 transitional cell carcinoma of the bladder. Urology 1999;53:308-313.

27 Cheng L, Weaver AL, Neumann RM, et al: Substaging of T1 bladder carcinoma based on the depth of invasion as measured by micrometer: a new proposal. Cancer 1999;86: 1035-1043.

28 van Rhijn BW, Liu L, Vis AN, et al: Prognostic value of molecular markers, sub-stage and European Organisation for the Research and Treatment of Cancer risk scores in primary T1 bladder cancer. BJU Int 2012;110:11691176.

29 Oosterlinck W, Lobel B, Jakse G, Malmström PU, Stöckle M, Sternberg C; European Association of Urology (EAU) Working Group on Oncological Urology: Guidelines on bladder cancer. Eur Urol 2002;41:105-112. 